

\section{Auf ein Wort...}

\section{Deutschland auf dem Weg zum Smart Government Jörn von Lucke}

Ausgehend von den Beiträgen von Hill (2016) und Schuppan/ Köhl (2016) zur Digitalisierung und Disruption im öffentlichen Sektor ist festzuhalten, dass die technische Entwicklung zwar oft evolutionär verläuft, die Wirkungen aber disruptiv sein können. Sorge bereitet, dass „Verwaltung 4.0“ als Begriff bereits zu verwässert ist und daher der Eindruck entstehen kann, es handele sich nur um die nächste Version von E-Government. Tatsächlich aber verbirgt sich dahinter ein echter technologischer Fortschritt im Internet der Dinge und Dienste, der sich womöglich durch Smart Government besser beschreiben lässt. Anhand der Häfler Definition von Smart Government wird aufgezeigt, wie Ansätze in der Tradition von Industrie 4.0 auf Staat und Verwaltung zu übertragen sind und welche Herausforderungen und Fragestellungen damit verbunden sein werden. Abschließend wird eine Forschungsagenda für Smart Government entwickelt, die staatliche Akteure dabei unterstützen kann, den Herausforderungen zu erkennen und dessen Potenzial zur Erfüllung öffentlicher Aufgaben zu nutzen.

\section{Lebenslagenorientiertes E-Government Sirko Hunnius, Tino Schuppan, Dirk Stocksmeier}

Ein wesentliches Ziel von E-Government ist es, den Verwaltungszugang für Bürger zu vereinfachen. Ein Schwerpunkt liegt dabei auf der Bündelung von Verwaltungsleistungen, beispielsweise anhand von Lebenslagen. Allerdings gibt es bislang kein allgemein akzeptiertes Set von Lebenslagen, das auf Basis einer transparenten Methode entwickelt wurde. Der vorliegende Beitrag entwickelt ein umfassendes und zugleich handhabbares Set von Lebenslagen. Damit soll die Komplexität für Bürger eingängig reduziert werden, was eine Orientierung in der Verwaltungswelt ermöglicht und gleichzeitig der gesamte Umfang des Leistungsportfolios der öffentlichen Verwaltung abgebildet werden. Auf Basis einer empirischen Analyse von online-Angeboten der Verwaltung werden 40 Lebenslagen gebildet, die das öffentliche Leistungsportfolio umfassend abbilden.

\section{Strukturen einer effizienten Wirtschaftsförderung Ralf-Rainer Piesold}

Wirtschaftsförderung gewinnt immer dann an Bedeutung, wenn die Finanzlage der Städte und Gemeinden von den politisch Verantwortlichen als schwierig eingestuft wird. Die finanzielle Situation vieler Städte und Gemeinden ist seit vielen Jahren sehr angespannt, so dass neben einer intensiven Überprüfung der Ausgaben nach Möglichkeiten zur Erhöhung der Einnahmen gesucht wird. Der kommunalen Wirtschaftsförderung wird hierbei besondere Aufmerksamkeit geschenkt. In diesem Aufsatz soll aufgezeigt werden, wie eine erfolgreiche und effiziente Wirtschaftsförderung gestaltet werden sollte. Durch die zunehmende Digitalisierung von Verwaltungsabläufen und die Verbesserung von E-GovernmentKonzepten wird eine Steigerung der Effektivität und Effizienz der Wirtschaftsförderung erreicht. In diesem Zusammenhang soll die Konzeption eines ganzheitlichen Ansatzes einer Wirtschaftsförderung zur Steigerung der Effektivität und Effizienz zur Diskussion gestellt werden.

\section{„Hauptstadt machen“ - Die Arbeitgebermarke für die Berliner Verwaltung Arne Richter}

Mit mittlerweile 3,5 Millionen Einwohnerinnen und Einwohnern ist Berlin ein attraktiver Standort für Wirtschaft, Wissenschaft,
Kultur oder Medien. Hinter der quirligen und stetig wachsenden Metropole Berlin steckt eine tatkräftige öffentliche Verwaltung. Berlin steht jedoch wie viele andere öffentliche Verwaltungen vor demografiebedingten Herausforderungen: Bis zum Jahr 2020 werden ca. 25 Prozent der Dienstkräfte altersbedingt ausscheiden, so dass ein massiver Einstellungs- und Nachbesetzungsbedarf besteht. Die Berliner Einstellungsbehörden stehen insbesondere mit den Behörden des Bundes und des Landes Brandenburg, aber auch mit der regionalen Privatwirtschaft in Konkurrenz. Um sich in dieser Konkurrenzsituation besser behaupten zu können und die Wahrnehmung der Berliner Verwaltung bei potenziellen Bewerberinnen und Bewerbern als attraktive Arbeitgeberin zu stärken, wurde eine einheitliche Arbeitgebermarke entwickelt.

\section{Controlling und Workflow-Management im Internationalen Tracing Service}

Volker Uhl

Der International Tracing Service (ITS), eine Einrichtung der Erinnerungskultur, hat das internationale Mandat, Überlebende und deren Familienangehörigen Auskunft zur Verfolgung im Nationalsozialismus zu geben. Die Einrichtung hat seit 2013 einen umfassenden Veränderungsprozess durchlaufen. Durch die ganzheitliche Bearbeitung von Suchanfragen in Teams wurde der Workflow umgestaltet und eine kleinteilige Bearbeitung nach dem Spezial-Know-how einzelner Mitarbeiter überwunden. Ergänzend wurde ein integrierter IT-basierter Workflow geschaffen, der die Neuorganisation der Arbeitsprozesse unterstützt.

\section{Systemische Organisationsentwicklung in Kommunen} Dino Schubert

Erfolgreiche organisationale Veränderungen stellen auch im öffentlichen Sektor die „Königsdisziplin“ dar. Die Herausforderung bei Veränderungsprozessen ist es, eine optimale Schnittmenge zwischen Mitarbeiterinteressen und Interesse der Dienststelle $\mathrm{zu}$ finden, damit Veränderungen überhaupt nachhaltige Akzeptanz bei den Umsetzenden finden. Dieser Artikel zeigt am Beispiel des systemischen Organisationsentwicklungsansatzes, wie dies gelingen kann. Er stellt den systemischen Ansatz am Fallstudienbeispiel eines ganzheitlichen Veränderungsprozesses der niedersächsischen Gemeindeverwaltung Bohmte dar.

EPSAS: Die Europäische Kommission beschleunigt das Tempo

Ralph Gerhards

Die Europäische Kommission macht Ernst. In der Entwicklung europäischer Rechnungslegungsstandards (EPSAS) für den öffentlichen Bereich geht die Europäische Union nunmehr den nächsten Schritt. Vor diesem Hintergrund betonen Nowak/ Rüdinger in ihrem Beitrag in Heft 5/2015 in Verwaltung \& Management die Notwendigkeit, die Frage der Konzeption von EPSAS in den Mittelpunkt zu rücken. Der Frage, ob mittels EPSAS überhaupt harmonisierte öffentliche Abschlüsse in der Europäischen Union und bessere Daten für die europäische Finanzstatistik möglich werden, gehen die Autoren nicht nach. Man mag dies bedauern. Letztlich aber tragen sie damit der Entwicklung auf EU-Ebene Rechnung. Dass es nicht mehr fraglich ist, ob es EPSAS geben wird, sondern nur noch, wie diese konzipiert werden sollen, wurde schon an anderer Stelle vermutet.

Nachrichten 